\title{
Histopathological features and prognostic significance of the micropapillary pattern in lung adenocarcinoma
}

\author{
Kazunori Kamiya ${ }^{1,2}$, Yuichiro Hayashi ${ }^{1}$, Junya Douguchi ${ }^{1}$, Akinori Hashiguchi ${ }^{1}$, \\ Taketo Yamada ${ }^{1}$, Yotaro Izumi ${ }^{2}$, Masazumi Watanabe ${ }^{2}$, Masafumi Kawamura ${ }^{2}$, \\ Hirohisa Horinouchi ${ }^{2}$, Naoki Shimada ${ }^{3}$, Koichi Kobayashi ${ }^{2}$ and Michiie Sakamoto ${ }^{1}$ \\ ${ }^{1}$ Department of Pathology, School of Medicine, Keio University, Tokyo, Japan; ${ }^{2}$ Department of Surgery, \\ Division of General Thoracic Surgery, School of Medicine, Keio University, Tokyo, Japan and ${ }^{3}$ Department of \\ Preventive Medicine and Public Health, School of Medicine, Keio University, Tokyo, Japan
}

\begin{abstract}
The micropapillary pattern is characterized by small papillary tufts with no fibrovascular core lying in spaces and has been reported as an aggressive variant of carcinoma in several organs. We investigated the histopathobiological properties of the micropapillary pattern with immunohistochemistry, serial sections, and electron microscopy in lung adenocarcinoma. We further analyzed its clinicopathological character and prognosis. The subjects included 383 adenocarcinoma cases, of which $184(48 \%)$ were micropapillary patternpositive and $199(52 \%)$ were micropapillary pattern-negative. On histology, micropapillary tufts seemed to float in the alveolar space or spaces encased by connective tissues, whereas serial sections revealed that most tufts had continuity with other tufts and even with the main tumor. Positive staining for the adhesion molecules $E$-cadherin and $\beta$-catenin suggested the preservation of tight adhesion, and electron microscopy showed the existence of intercellular junctions. Negative staining for laminin and loss of basement membrane as determined by electron microscopy suggest a loss of cell-matrix contact. Positive staining for Ki-67 indicates that cells constituting micropapillary tufts retained their proliferation potency. There were no CD34-positive cells in micropapillary tufts, and the loss of the vascular core was confirmed. In micropapillary pattern-positive cases, lymphatic invasion was identified significantly more frequently than in micropapillary pattern-negative cases $(P<0.001)$, even at stagelA (without lymph node metastasis, $N=197)(P<0.001)$. The 5-year and 10-year overall survival rates of the micropapillary pattern-positive stagelA group were 77.6 and $67.6 \%$, respectively, which were significantly less than those of the micropapillary pattern-negative stagelA group (98.1 and $98.1 \%)(P=0.001)$. In conclusion, cells constituting the micropapillary pattern are likely to have acquired anchorage-independent growth and a potential for high malignancy.

Modern Pathology (2008) 21, 992-1001; doi:10.1038/modpathol.2008.79; published online 30 May 2008
\end{abstract}

Keywords: lung adenocarcinoma; micropapillary pattern; histopathology; prognosis; immunohistochemistry; electron microscopy

\section{Introduction}

Lung cancer is one of the most common causes of cancer-related death worldwide. ${ }^{1}$ The prognosis of patients with lung cancer is generally poor, and the overall 5 -year survival rate is $15 \% .^{2}$ Of the four main histological types of lung cancer, adenocarcinoma is increasing in frequency and accounts for almost half of all lung cancers. ${ }^{3}$ Recent advances in diagnostic

Correspondence: Dr M Sakamoto, MD, Department of Pathology, School of Medicine, Keio University, 35 Shinano-machi, Shinjyuku-ku, Tokyo 160-8582, Japan.

E-mail: msakamot@sc.itc.keio.ac.jp

Received 03 January 2008; revised 16 April 2008; accepted 17 April 2008; published online 30 May 2008 imaging have enhanced the capability for diagnosis at the early stage, and histopathological studies indicate the existence of good prognostic groups in lung cancer. Bronchioloalveolar carcinoma, defined as a non-invasive subtype of lung adenocarcinoma, ${ }^{3}$ is recognized as ground-glass opacity lesion on computed tomography and is reported to have a good prognosis. ${ }^{4}$ However, the overall 5-year survival of patients with stageIA (T1N0M0: T1, tumor greatest dimension $\leq 30 \mathrm{~mm}$ and surrounded by pleura; No, no regional lymph node metastasis; M0, no distant metastasis) ${ }^{5}$ adenocarcinomas does not reach $80 \%,{ }^{6,7}$ which indicates the existence of a poor prognostic group. Factors including smoking history, serum level of carcinoembryonic antigen, and tumor size, as well as lymphatic and venous 
invasion have been identified as poor prognostic factors for lung adenocarcinoma. ${ }^{8-12}$

Recently, a pathological entity called the micropapillary pattern has been reported to have worse outcomes in breast, ${ }^{13}$ colon, ${ }^{14}$ urinary tract, ${ }^{15}$ ovary, ${ }^{16}$ salivary gland, ${ }^{17}$ and lung ${ }^{18,19}$ cancer. The micropapillary pattern in lung adenocarcinoma is characterized by small papillary tufts lying in alveolar spaces or in spaces encased by connective tissues, with the tufts having no fibrovascular core, ${ }^{18,19}$ and it has been reported as an important factor for poor prognosis. ${ }^{18-21}$ However, the detailed histopathobiological features of the micropapillary pattern have not yet been clarified. We investigated the histological, immunohistochemical, electron microscopic, and clinicopathological properties of the micropapillary pattern to elucidate why it could be a factor in poor prognoses.

\section{Materials and methods}

\section{Surgical Specimens and Patient Characteristics}

From January 1993 to December 2006 in Keio University Hospital, 526 patients underwent complete surgical resection of lung cancer diagnosed as primary lung adenocarcinoma. We reviewed these sections macroscopically and microscopically as mentioned below. The histological type of the tumor was described according to the World Health Organization classification ${ }^{3}$ and pathological staging was performed according to the classification of the Union Internationale Coutre le Cancer. ${ }^{5}$ Because bronchioloalveolar carcinoma has been defined as a non-invasive lesion ${ }^{3}$ and there was no existence of the micropapillary pattern in cases composed only of bronchioloalveolar carcinoma $(N=68)$, we excluded these cases from clinicopathological analysis. For accuracy of survival analysis, stageIIIB and stageIV cases with the possibility of incomplete resection were excluded as well. Therefore, the final population for clinicopathological analysis consisted of 383 cases (214 men and 169 women ranging from 29 to 85 years of age, with an average age of 62.3 years). Clinicopathological information was obtained by reviewing the medical charts in detail with regard to age $(<60$ or $\geq 60$ years), sex (male or female), smoking history (nonsmoker or smoker: smoker was defined as more than 1 year of smoking history), recurrence, and survival. The follow-up period ranged from 3 to 158 months (mean was 60.9 months). This study was conducted under the approval of the Ethics Committee of the Keio University School of Medicine.

\section{Histological Examination}

Surgically resected specimens were fixed routinely in $10 \%$ formalin, cut serially into 5 - to 7 -mm-thick slices, and macroscopically examined. From the section including the maximum tumor diameter, all the tumor tissues as well as the surrounding lung tissue were removed and embedded in paraffin, and then cut into 4- $\mu \mathrm{m}$-thick sections. Finally, hematoxylin-eosin and Elastica Van Gieson staining were performed and all sections for each patient were observed. The following histopathological factors were evaluated: tumor size (maximum tumor diameter $\leq 30$ or $>30 \mathrm{~mm}$ ), lymph node metastasis, pleural invasion, lymphatic invasion, and venous invasion. The histological patterns were divided into four distinctive subtypes: bronchioloalveolar carcinoma as well as acinar, papillary, and solid adenocarcinoma with mucin; the latter three subtypes were defined as invasive. ${ }^{3}$ The dominant subtype of each tumor was then documented.

The micropapillary pattern was defined according to the two previous studies; ${ }^{18,19}$ it was identified as small tufts lying in alveolar spaces or in spaces encased within thin walls of connective tissue, and the tufts had no fibrovascular core. The extent of the micropapillary pattern was subclassified as none ( $0 \%$ of the tumor), focal $(<10 \%)$, moderate $(<50 \%)$, or extensive $(\geq 50 \%)$ based on the proportion of micropapillary pattern area in the tumors.

Furthermore, three micropapillary pattern-positive samples (extent: moderate and extensive) were selected and 100 serial sections stained by hematoxylin-eosin were then examined sequentially at intervals of $12 \mu \mathrm{m}$ with attention paid to the morphology of the micropapillary pattern.

\section{Immunohistochemistry}

For immunohistochemistry, 20 cases of tumor with the micropapillary pattern were randomly selected and studied (10 cases with lymph node metastasis and 10 without).

Four-micrometer-thick sections were deparaffinized, rehydrated, and incubated in $0.03 \% \mathrm{H}_{2} \mathrm{O}_{2}$ in $95 \%$ methanol at room temperature for $20 \mathrm{~min}$ to block the endogenous peroxidase activity. Autoclave pretreatment at $120^{\circ} \mathrm{C}$ for $10 \mathrm{~min}$ in $10 \mathrm{mM}$ citrate buffer ( $\mathrm{pH}$ 6.0) was used for E-cadherin and Ki-67 antigen retrieval. Water bath pretreatment at $100^{\circ} \mathrm{C}$ for $10 \mathrm{~min}$ in $10 \mathrm{mM}$ citrate buffer (pH 9.0) was used for $\beta$-catenin and CD34 antigen retrieval. Incubation in $4 \mathrm{mg} / \mathrm{ml}$ pepsin (Dako, Glostrup, Denmark) in $0.2 \mathrm{~N} \mathrm{HCl}$ at $37^{\circ} \mathrm{C}$ for $60 \mathrm{~min}$ was used for laminin antigen retrieval. All sections were incubated for 20 min with normal horse serum to eliminate nonspecific staining and then incubated with the following primary antibodies: anti-human E-cadherin (1:200; Novocastra Laboratories, Newcastle, UK), anti-human $\beta$-catenin (1:200; Santa Cruz Technology, Santa Cruz, CA, USA), anti-human laminin (1:400; Dako), anti-human CD34 (1:100, Dako), and anti-human Ki-67 (diluted at 1:400; Dako). These antibodies were applied overnight at $4{ }^{\circ} \mathrm{C}$, followed by incubation with the secondary antibody 
(ImmPRESS $^{\mathrm{TM}}$ Reagent Kit, Vector Laboratories, Burlingame, CA, USA) for $30 \mathrm{~min}$. Slides were then incubated in $\mathrm{DAB} /$ Tris solution (three $\mathrm{DAB} /$ Tris tablets diluted in $150 \mathrm{ml}$ distilled water; Muto Pure Chemicals, Tokyo, Japan) supplemented with $15 \mu \mathrm{l}$ of $30 \% \mathrm{H}_{2} \mathrm{O}_{2}$. Finally, the slides were counterstained with hematoxylin. Ki-67 expression was evaluated by counting 400 tumor cells in 4 high-power fields $(\times 200)$ from the micropapillary pattern areas in all 20 cases, and expressed as the percentage nuclearstained cells.

\section{Electron Microscopy}

Three tumor specimens, already fixed with formalin and diagnosed as adenocarcinoma with the micropapillary pattern (extent: moderate and extensive), were selected and studied. Specimens were refixed in $2.5 \%$ glutaraldehyde in an isomolar phosphate buffer ( $\mathrm{pH}$ 7.4), postfixed in 1\% osmium tetroxide for $1.5 \mathrm{~h}$, dehydrated in graded alcohol, and embedded in Epon 812. Ultrathin sections were mounted on copper grids, stained with uranyl acetate and lead citrate, and then examined with a JEM-1200 transmission electron microscope (Nihon Denshi, Tokyo, Japan).

\section{Statistical Analysis}

The length of disease-free survival was defined as the period from the date of surgery to the date when recurrence was diagnosed, and that of overall survival was defined as the period until the date of either death as a result of the tumor or the most recent follow-up. Survival curves were plotted using the Kaplan-Meyer method and $P$-values were calculated using the log-rank test. The correlations between several clinicopathological characteristics and histological subgroups were evaluated using the $\chi^{2}$ test. $P$-values of less than 0.05 were accepted as significant. Data were analyzed with Statistical Standard Package Service Solution software (SPSS for Standard version 15.0; SPSS Inc, Chicago, IL, USA).

\section{Results}

\section{Histological Examination}

Of the 383 cases of adenocarcinoma, 103 (27\%), 69 $(18 \%), 182(48 \%)$, and $29(7 \%)$ were in the dominant bronchioloalveolar carcinoma, acinar, papillary, and solid with mucin subtypes, respectively (Table 1).

On histology, micropapillary tufts had no fibrovascular core and were detected in the alveolar space (Figure 1a), or in spaces inside the tumor that in most cases represented retracted connective tissue (Figure 1b), and adjacent to any of the above-mentioned four histological pattern subtypes.
The micropapillary pattern was detected in 184 patients (109 men and 75 women), with $19(11 \%)$, $33(18 \%), 117(63 \%)$, and $15(8 \%)$ in the dominant bronchioloalveolar carcinoma, acinar, papillary, and solid with mucin subtypes, respectively (Table 1).

The extent of the micropapillary pattern present in tumors varied. The numbers of patients with focal, moderate, and extensive were 65 (35\%), 85 $(46 \%)$, and $34(19 \%)$, respectively.

In the three cases studied using serial sections, we found that most tufts had continuity with other tufts and main tumor. For example, a tuft marked by $\Rightarrow$ ) in section 12 was consecutive with another tuft in section 8 and with the main tumor in section 1. Two isolated tufts, identified by $(\rightarrow)$ in section 4 , were shown to be consecutive in section 10. There also existed several tufts lacking continuity with any other tuft. The tuft marked by $(\rightarrow)$ in sections 5,6 , and 7 could not be confirmed to be continuous with any other tuft (Figure 2). Micropapillary tufts were observed to present an intricate morphology.

\section{Immunohistochemical Findings}

E-cadherin was positive at intercellular cell membranes in every micropapillary tuft, and showed no difference in staining intensity when compared with neoplastic cells in the main tumor (Figure 3a). $\beta$-Catenin staining showed the same pattern as E-cadherin (Figure 3b). Tight adhesion of cells constituting micropapillary tufts was therefore believed to be maintained. Laminin was identified in the basement membrane of normal alveolar cells and neoplastic cells of the main tumor, but on the other hand, it was not found in any cell constituting micropapillary tufts (Figure 3c). It is most likely that cells constituting micropapillary tufts had no basement membrane and therefore showed disordered continuity with the matrix. Cells constituting micropapillary tufts appeared to pile up. CD34-positive cells were enriched in the stroma of the main tumor and in the alveolar septum, whereas no CD34positive cells were found in any micropapillary tuft (Figure 3d). No vascularity was observed in micropapillary tufts. These findings were confirmed in all 20 cases. Differences in staining pattern and intensity between cases with and without lymph node metastasis ( $N=10$ each) were not found. To determine whether cells composing micropapillary tufts had proliferation potency, Ki-67 staining was performed. Cells constituting micropapillary tufts showed positive staining for Ki-67 (Figure 3e); positive rates ranged from 5 to $45 \%$ with a mean of $20.3 \%$. Cells constituting micropapillary tufts were therefore believed to have proliferation potency.

\section{Electron Microscopy}

Electron microscopy showed no basement membrane or vascular structures. Microvilli were some- 
Table 1 Correlations between clinicopathological characteristics and the micropapillary pattern $(N=383)$

\begin{tabular}{|c|c|c|c|c|}
\hline \multirow[t]{2}{*}{ Characteristics } & \multirow[t]{2}{*}{ No. of cases } & \multicolumn{2}{|c|}{ Micropapillary pattern } & \multirow[t]{2}{*}{$\mathrm{P}$-value } \\
\hline & & Negative (\%) & Positive (\%) & \\
\hline \multicolumn{5}{|l|}{ Gender } \\
\hline Male & 214 & $105(53)$ & $109(59)$ & \\
\hline Female & 169 & $94(47)$ & $75(41)$ & 0.202 \\
\hline \multicolumn{5}{|l|}{ Age (years) } \\
\hline$<60$ & 127 & $60(30)$ & $67(36)$ & \\
\hline$\geq 60$ & 256 & $139(70)$ & $117(64)$ & 0.193 \\
\hline \multicolumn{5}{|l|}{ Smoking } \\
\hline Nonsmoker & 185 & $107(54)$ & $80(44)$ & \\
\hline Smoker & 198 & $92(46)$ & $104(56)$ & 0.044 \\
\hline \multicolumn{5}{|c|}{ Maximum tumor diameter (mm) } \\
\hline$\leq 30$ & 261 & $139(70)$ & $122(66)$ & \\
\hline$>30$ & 122 & $60(30)$ & $62(34)$ & 0.457 \\
\hline \multicolumn{5}{|l|}{ Lymph node metastasis } \\
\hline Negative & 267 & $164(82)$ & $103(56)$ & \\
\hline Positive & 126 & $35(18)$ & $81(44)$ & $<0.001$ \\
\hline \multicolumn{5}{|l|}{ Pleural invasion } \\
\hline Negative & 265 & $150(75)$ & $115(63)$ & \\
\hline Positive & 118 & $49(25)$ & $69(37)$ & 0.006 \\
\hline \multicolumn{5}{|l|}{ Lymphatic invasion } \\
\hline Negative & 215 & $154(77)$ & $61(33)$ & \\
\hline Positive & 168 & $45(23)$ & $123(67)$ & $<0.001$ \\
\hline \multicolumn{5}{|l|}{ Venous invasion } \\
\hline Negative & 309 & $182(92)$ & $127(69)$ & \\
\hline Positive & 74 & $17(8)$ & $57(31)$ & $<0.001$ \\
\hline \multicolumn{5}{|c|}{ Dominant histological subtype } \\
\hline Bronchioloalveolar & 103 & $84(42)$ & $19(11)$ & \\
\hline Non-bronchioloalveolar & 280 & $115(58)$ & $165(89)$ & $<0.001$ \\
\hline Acinar & 69 & $36(18)$ & $33(18)$ & \\
\hline Non-acinar & 314 & $163(82)$ & $151(82)$ & 0.968 \\
\hline Papillary & 182 & $65(33)$ & $117(63)$ & \\
\hline Non-papillary & 201 & $134(67)$ & $67(37)$ & $<0.001$ \\
\hline Solid with mucin & 29 & $14(7)$ & $15(8)$ & \\
\hline Non-solid with mucin & 354 & $185(93)$ & $169(92)$ & 0.680 \\
\hline
\end{tabular}

times observed but their distribution was focal and random. The apical and basal sides of the cells were not clearly delineated (Figure 4a), and showed intercellular junctions in micropapillary tufts (Figure 4b). These findings were confirmed in all three cases. Some cells constituting micropapillary tufts appeared to accumulate without cell polarity.

\section{Correlation of Micropapillary Pattern and Clinicopathological Factors}

Clinicopathological characteristics of patients and their tumors were compared between micropapillary pattern-positive (with focal, moderate, and extensive extent as mentioned below) and micropapillary pattern-negative groups (Table 1). Smoking $(P=0.044)$, lymph node metastasis
$(P<0.001)$, pleural invasion $(P=0.006)$, lymphatic invasion $(P<0.001)$, venous invasion $(P<0.001)$, dominant non-bronchioloalveolar carcinoma subtype $(P<0.001)$, and dominant papillary subtype $(P<0.001)$ were significantly associated with the micropapillary pattern. Especially strong correlations between the micropapillary pattern and lymph node metastasis, lymphatic invasion, and dominant non-bronchioloalveolar carcinoma subtype were observed. There was no association with gender $(P=0.202)$, age $(P=0.193)$, or tumor size $(P=0.457) . \quad$ In stageIA cases $(N=197)$, smoking $(P=0.031)$, lymphatic invasion $(P<0.001)$, venous invasion $(P<0.001)$, dominant non-bronchioloalveolar carcinoma subtype $(P<0.001)$, and dominant papillary subtype $(P<0.001)$ were significantly associated with the micropapillary pattern (Table 2). 

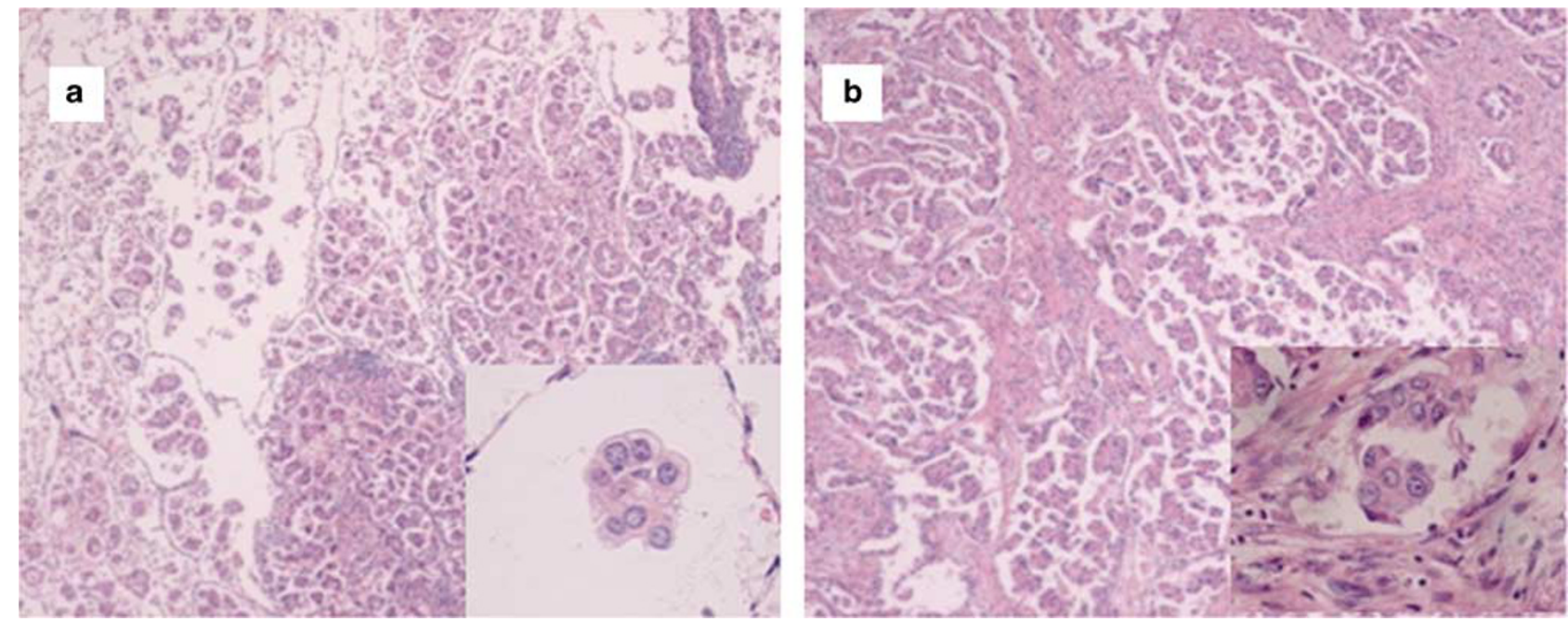

Figure 1 Representative histology of the micropapillary pattern. (a and b) The micropapillary pattern was characterized by small tufts with no fibrovascular core (a) lying in the alveolar space or (b) in spaces that in most cases represented retracted connective tissue. (a and b) Hematoxylin-eosin: $\times 40$; inset: $\times 400$.
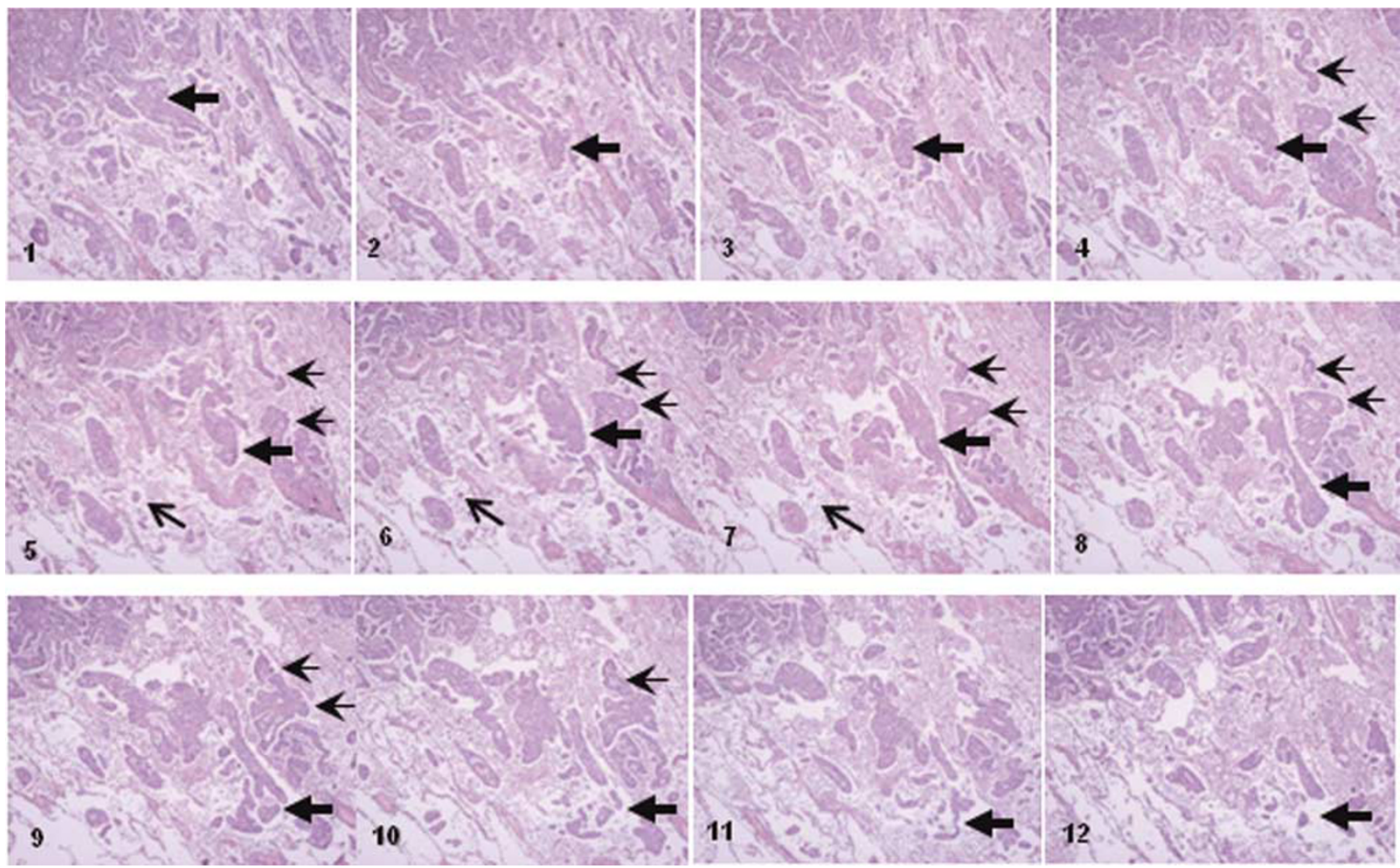

Figure 2 Serial sections of a micropapillary pattern-positive specimen. The tuft marked by

) in section 12 is consecutive with another tuft in section 8 and even with the main tumor in section 1. Two isolated tufts identified by $(\rightarrow$ ) in section 4 are shown to be consecutive in section 10 . The tuft identified by $(\rightarrow)$ in sections 5,6 , and 7 could not be identified as continuous with any other tuft. Sections 1-16 hematoxylin-eosin at $\times 40$.

\section{Prognostic Significance of the Micropapillary Pattern}

Survival curves showed that as the extent of the micropapillary pattern progressed, the prognosis tended to worsen, and that the disease-free and overall survival for the focal group were worse than in the none group $(P=0.027$ and $P=0.068)$ (Figure $5 a$ and $b$ ). In this study, cases in the none group $(199,52 \%)$ were therefore classified as micropapillary pattern-negative and the remainder of cases $(184,48 \%)$ were classified as micropapillary pattern-positive. Disease-free and overall survival for 

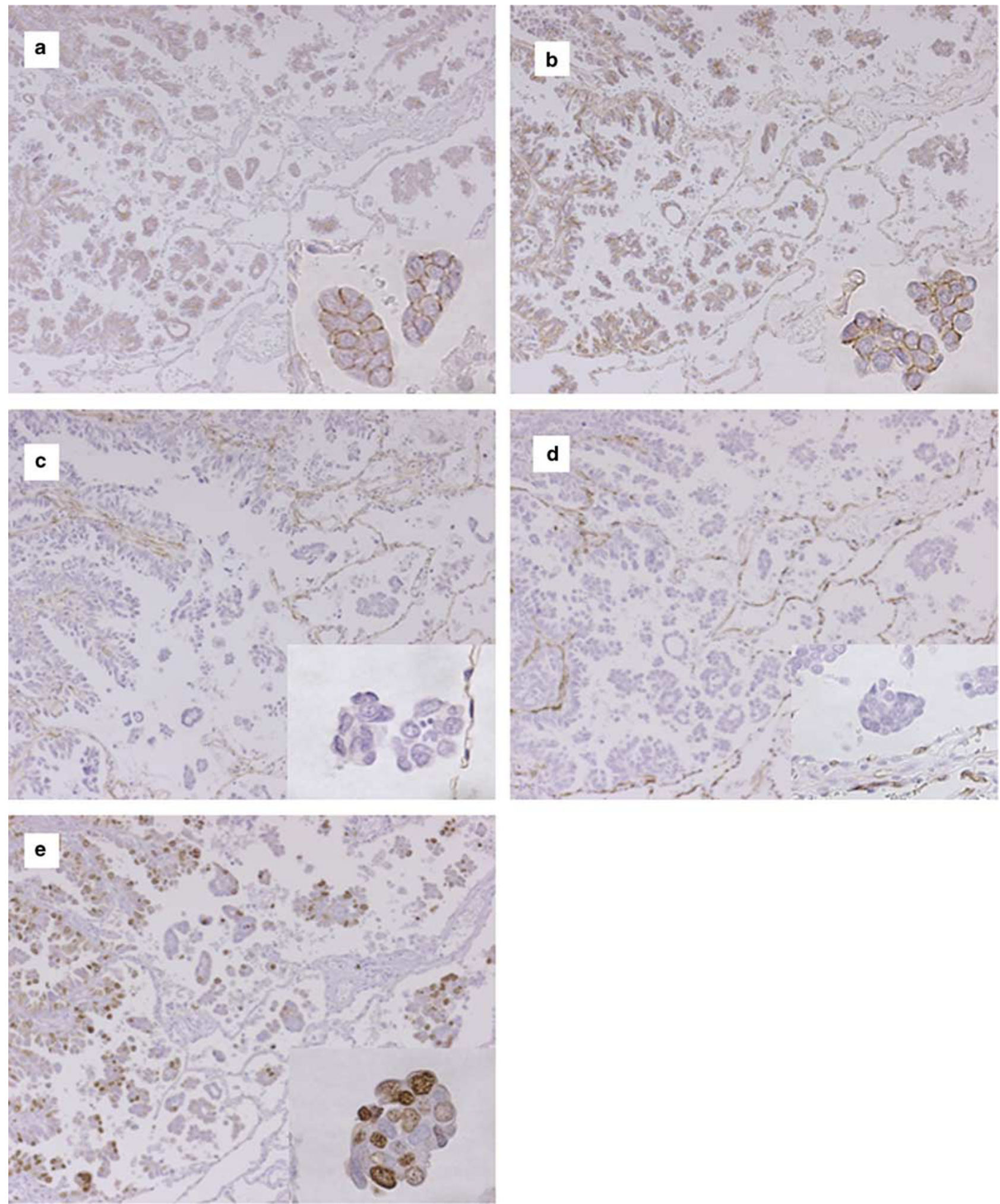

Figure 3 Immunohistochemical findings. (a) E-cadherin and (b) $\beta$-catenin were expressed at intercellular cell membranes of micropapillary tufts. (c) Laminin was found in the basement membrane of normal alveolar cells and in neoplastic cells in the main tumor, but was not found in cells constituting micropapillary tufts. (d) CD34-positive cells were enriched in the stroma of main tumor and the alveolar septum, whereas no CD34-positive cells were found in micropapillary tufts. (e) Cells constituting micropapillary tufts stained positive for Ki-67. (a) E-cadherin (b) $\beta$-catenin (c) Laminin (d) CD34 (e) Ki-67 × 40; inset: $\times 400$. 

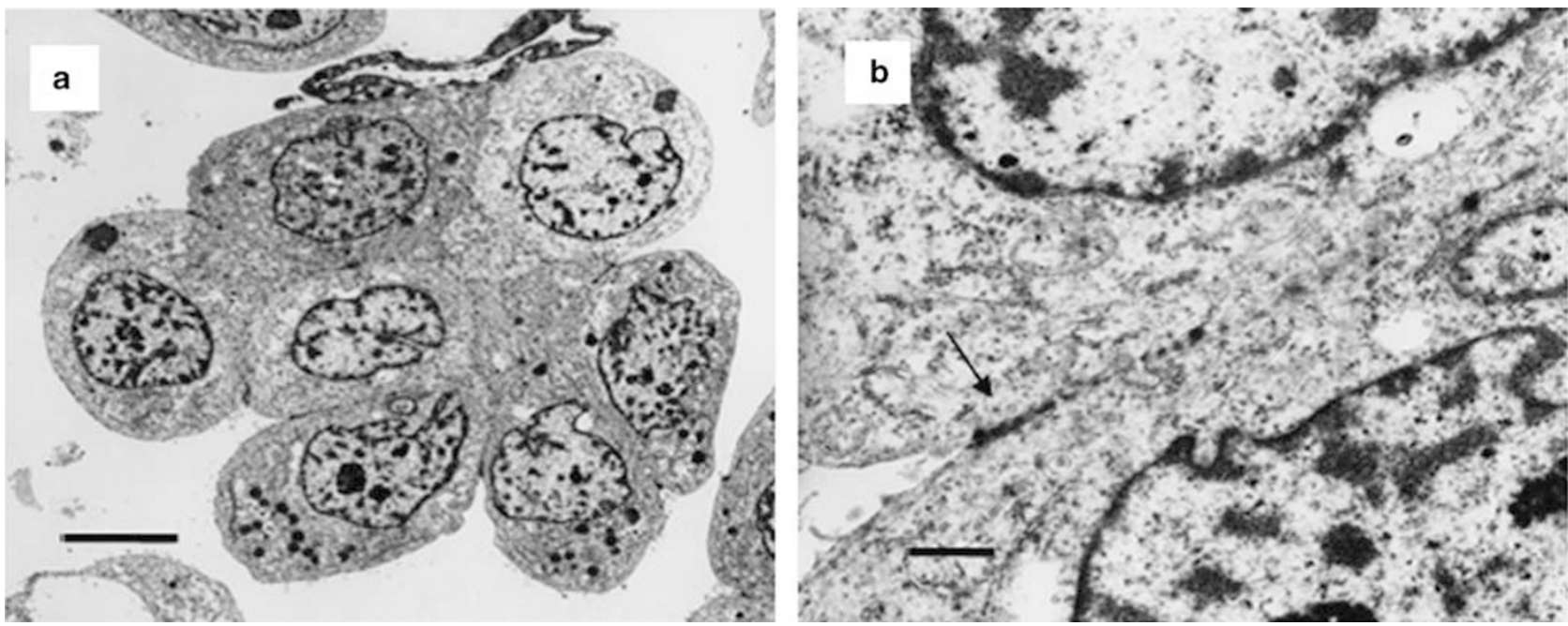

Figure 4 Electron microscopic findings. (a) No basement membrane or vascular structures were observed. Microvilli distribution was focal and random, and the apical and basal sides could not be clearly identified. (b) Intercellular junctions (arrow). (a) Scale bar $=5 \mu \mathrm{m}$; (b) scale bar $=500 \mathrm{~nm}$.

Table 2 Correlation between clinicopathological characteristics and the micropapillary pattern in stageIA patients $(N=197)$

\begin{tabular}{|c|c|c|c|c|}
\hline \multirow[t]{2}{*}{ Characteristics } & \multirow[t]{2}{*}{ No. of cases } & \multicolumn{2}{|c|}{ Micropapillary pattern } & \multirow[t]{2}{*}{$\mathrm{P}$-value } \\
\hline & & Negative (\%) & Positive (\%) & \\
\hline \multicolumn{5}{|l|}{ Gender } \\
\hline Male & 108 & $60(51)$ & $48(62)$ & \\
\hline Female & 89 & $59(49)$ & $30(38)$ & 0.125 \\
\hline \multicolumn{5}{|l|}{ Age (years) } \\
\hline$<60$ & 68 & $36(30)$ & $32(41)$ & \\
\hline$\geq 60$ & 129 & $83(70)$ & $46(59)$ & 0.120 \\
\hline \multicolumn{5}{|l|}{ Smoking } \\
\hline Nonsmoker & 107 & $72(60)$ & $35(45)$ & \\
\hline Smoker & 90 & $47(40)$ & $43(55)$ & 0.031 \\
\hline \multicolumn{5}{|l|}{ Lymphatic invasion } \\
\hline Negative & 157 & $111(93)$ & $46(59)$ & \\
\hline Positive & 40 & $8(7)$ & $32(41)$ & $<0.001$ \\
\hline \multicolumn{5}{|l|}{ Venous invasion } \\
\hline Negative & 180 & 116 (98) & $64(82)$ & \\
\hline Positive & 17 & $3(2)$ & $14(18)$ & $<0.001$ \\
\hline \multicolumn{5}{|c|}{ Dominant histological subtype } \\
\hline Bronchioloalveolar & 79 & $64(54)$ & $15(19)$ & \\
\hline Non-bronchioloalveolar & 118 & $55(46)$ & $63(81)$ & $<0.001$ \\
\hline Acinar & 28 & $17(14)$ & $11(14)$ & \\
\hline Non-acinar & 169 & $102(86)$ & $67(86)$ & 0.971 \\
\hline Papillary & 80 & 34 (27) & $46(59)$ & \\
\hline Non-papillary & 117 & $85(73)$ & $32(41)$ & $<0.001$ \\
\hline Solid with mucin & 10 & $4(3)$ & $6(8)$ & \\
\hline Non-solid with mucin & 187 & $115(97)$ & $72(92)$ & 0.176 \\
\hline
\end{tabular}

the micropapillary pattern-positive group were significantly worse than for the micropapillary pattern-negative group $(P<0.001$ and $P=0.027)$.

In all four dominant subtype groups, disease-free and overall survival rates for the micropapillary pattern-positive group tended to be worse than those for the micropapillary pattern-negative group (Figure 5c and d). The micropapillary pattern was therefore considered to affect on prognosis irrespective of dominant histological subtype. 

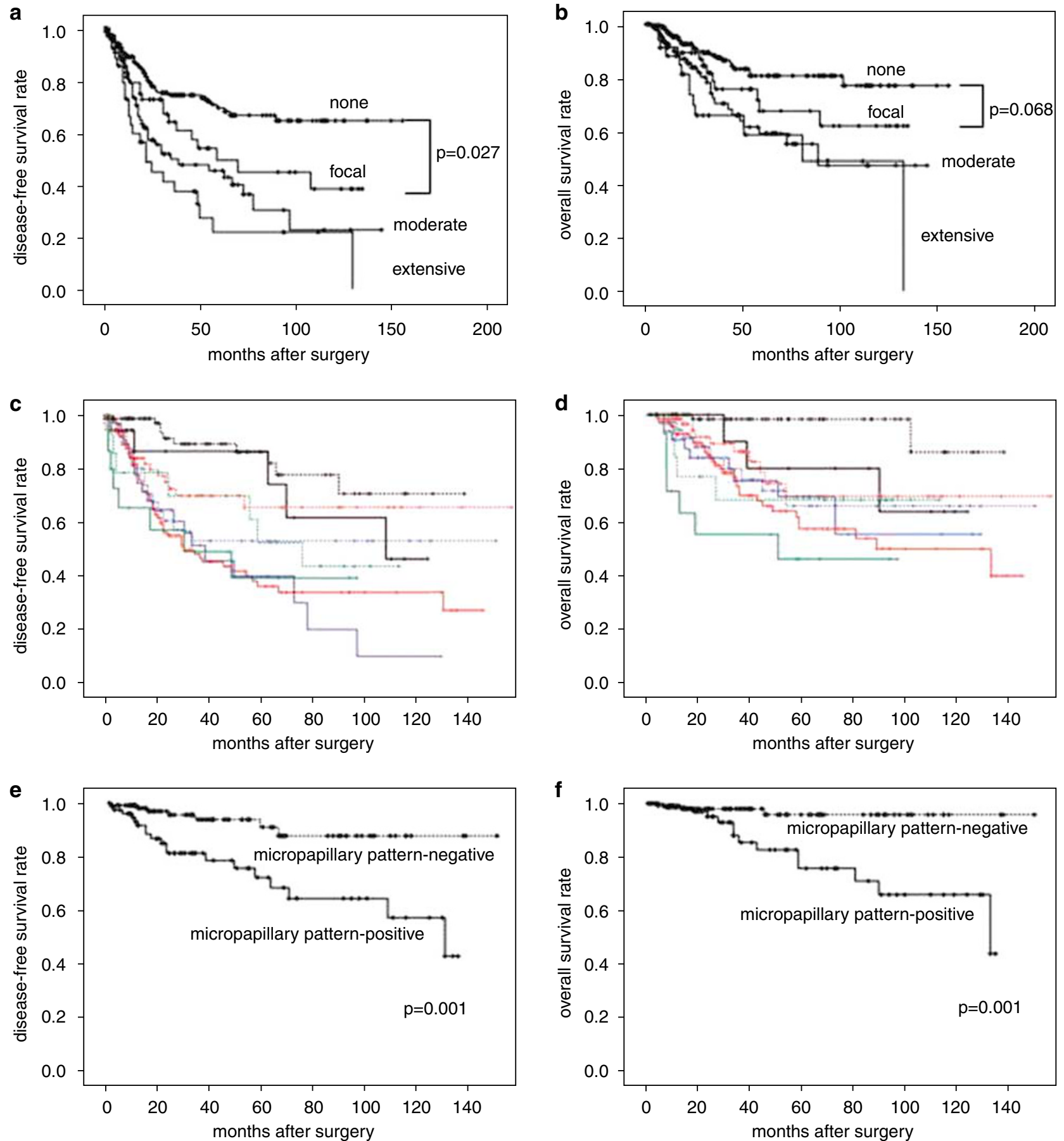

Figure 5 (a and b) Survival curves with different extents of the micropapillary pattern $(N=383)$. The disease-free and overall survival of the focal group $(N=65)$ were worse than those of the none group $(N=199)(P=0.027$ and $P=0.068)$. (c and d) Survival curves grouped by the presence of the micropapillary pattern and dominant histological subtype. Black, blue, red, and green lines show dominant bronchioloalveolar carcinoma $(N=103)$, acinar $(N=69)$, papillary $(N=182)$, and solid with mucin subtype $(N=29)$, respectively. Solid and dotted lines show micropapillary pattern-positive and pattern-negative, respectively. No significant differences in survival between dominant acinar, papillary, and solid with mucin carcinomas were found. The difference between micropapillary pattern-positive and pattern-negative carcinomas was significant in the dominant papillary subtype (red) for disease-free survival $(P=0.008)$ and in the dominant bronchioloalveolar carcinoma subtype (black) for overall survival $(P=0.046)$. Differences were observed in other subtypes, but they were small and not significant. (e and f) The 5-year and 10-year overall survival rates of the micropapillary pattern-positive group $(N=78)$ were 77.6 and $67.6 \%$, respectively, which were significantly worse than those of the micropapillary pattern-negative group $(N=119)$ (98.1 and $98.1 \%)$ in stageIA.

We then analyzed whether the micropapillary pattern had prognostic significance in the early stage when lymph node metastasis was absent at the time of surgery. At stageIA, disease-free and overall survival rates for the micropapillary pattern-positive group $(N=119)$ were significantly worse than for the 
micropapillary pattern-negative group $(N=78)$ $(P=0.001$ and $P=0.001)$ (Figure $5 \mathrm{e}$ and $\mathrm{f})$. The 5 -year and 10-year overall survival rates of the micropapillary pattern-positive group in stageIA were 77.6 and $67.6 \%$, respectively, which were significantly worse than those of the micropapillary pattern-negative group (98.1 and $98.1 \%$ ).

\section{Discussion}

On histology, micropapillary tufts were isolated from the main tumor and seemed to float in airspaces. Using serial sections, we identified an intricate structure of the micropapillary pattern in which the tufts appeared to extend into airspaces. Compared with other organs, the lung contains sufficient airspaces that cells constituting micropapillary tufts might extend easily and extensively beyond the main tumor. On histological diagnosis, it may therefore be difficult to discriminate the micropapillary pattern from intrapulmonary metastasis. However, some vascularities or stroma are normally found in intrapulmonary metastatic lesions, but not in micropapillary tufts. Both forms should be distinguished clearly. Because histopathological findings showed no vascularity in micropapillary tufts, the route of nourishment for their constitutive cells is uncertain. However, nutrients might be supplied from the surrounding epithelial lining fluids in the alveolar space.

Luna-More et $a l^{13}$ suggested that neoplastic cells in invasive micropapillary carcinoma of the breast display reverse polarity. We explored the possibility that some of the cells constituting micropapillary tufts in lung adenocarcinomas also lost their cell polarity. Tufts composed of such cells are not micro 'papillary' but rather micro 'cluster.' Igaki et $a l^{22}$ described that the loss of polarity in the presence of oncogenic Ras resulted in accelerated tumor invasion through the association of Ras and c-Jun N-terminal kinase pathway activation in Drosophila. The micropapillary pattern is believed to possess intense invasive potency considering its correlation with lymphatic, venous invasion. Loss of cell polarity might be related to this potency.

Loss of vascularity and cell-matrix contact, as well as the preservation of intercellular junctions in micropapillary tufts were demonstrated in this study. The micropapillary pattern is believed to represent the morphological piling up of neoplastic cells. Normally, when displaced from the extracellular matrix, epithelial cells undergo apoptosis (anoikis). ${ }^{23}$ In neoplastic cells, alterations in the expression of cell-matrix adhesion molecules, integrins, integrin-associated signaling molecules, or apoptosis regulators can lead to anoikis resistance. ${ }^{23-24}$ In the present study, we further revealed that cells constituting micropapillary tufts had proliferation potency using Ki-67 staining, and also revealed a strong correlation between the micropapillary pattern and lymphatic invasion and metastasis based on clinicopathological analysis. These cells have most likely acquired anoikis resistance and facilitated anchorage-independent growth, which are advantageous for proliferation during lymphatic cancer metastasis. The micropapillary pattern is a distinct histobiological feature that may therefore help to elucidate the mechanism of lymphatic cancer metastasis.

Our data show that survival for the focal group was worse than for the none group, and also show that as the extent of the micropapillary pattern progressed, the prognosis tended to worsen. Makimoto et $a l^{21}$ reported no significant difference in survival between the focal (extent: $<10 \%$ ) group and the none group, but the criteria for the extent of the micropapillary pattern and the methods used to evaluate it have been inconsistent. ${ }^{20}$

We clearly showed that in stageIA when lymph node metastasis was absent at the time of surgery, the 5-year and 10-year overall survival rates of the micropapillary pattern-negative group were high (98.1 and $98.1 \%$ ), whereas those of the micropapillary pattern-positive group were much lower (77.6 and $67.6 \%$ ). Currently, therapeutic decisions are mainly based on the TNM staging of cancer. The micropapillary pattern could be a significant prognostic factor even in the early stage.

In conclusion, the micropapillary pattern in lung adenocarcinoma is a distinct histopathological variant with biological and prognostic significance. This entity should be recognized carefully on pathological diagnosis.

\section{Acknowledgement}

This work was supported in part by a Grant-in-Aid for Scientific Research on Priority Areas 'Cancer' and for the 21st Century Center of Excellence program from the Ministry of Education, Science, Sports and Culture of Japan, for the Third Term Comprehensive 10-Year Strategy for Cancer Control from the Ministry of Health and Welfare of Japan. We thank Yuko Hashimoto, Naomichi Yagi, and Hiroshi Suzuki for technical assistance.

\section{References}

1 Alberg AJ, Samet JM. Epidemiology of lung cancer. Chest 2003;123(1 Suppl):21S-49S.

2 Jemal A, Siegal R, Ward E, et al. Cancer statistics. CA Cancer J Clin 2006;56:10-30.

3 Travis WD, Brambilla E, Muller-Hermelink HK, et al. World Health Organization Classification of Tumors: Pathology and Genetics of Tumours of Lung, Pleura, Thymus and Heart. IARC Press: Lyon, 2004, pp 26-34.

4 Noguchi M, Morikawa A, Kawasaki M, et al. Small adenocarcinoma of the lung. Histologic characteristics and prognosis. Cancer 1995;75:2844-2852. 
5 Sobin LH, Wittenkind CH, (eds). TNM Classification of Malignant Tumours, 6th edn. Wiley-Liss: New York, 2002.

6 Beadsmoore CJ, Screaton NJ. Classification, staging and prognosis of lung cancer. Eur J Radiol 2003;45: 8-17.

7 Goya T, Asamura H, Yoshimura H, et al. Prognosis of 6644 resected non-small cell lung cancers in Japan: a Japanese Lung Cancer Registry Study. Lung Cancer 2005;50:227-234.

8 Kawai H, Tada A, Kawahara M, et al. Smoking history before surgery and prognosis in patients with stageIA non-small-cell lung cancer-a multicenter study. Lung Cancer 2005;49:63-67.

9 Sakao Y, Tomimitsu S, Takeda Y, et al. Carcinoembryonic antigen as a predictive factor for postoperative tumor relapse in early-stage lung adenocarcinoma. Eur J Cardiothorac Surg 2004;25:520-522.

10 Gajra A, Newman N, Gamble GP, et al. Impact of tumor size on survival in stageIA non-small cell lung cancer: a case for subdividing stageIA disease. Lung Cancer 2003;42:51-57.

11 Goldstein NS, Mani A, Chmielewski G, et al. Prognostic factors in T1 No M0 adenocarcinomas and bronchioloalveolar carcinomas of the lung. Am J Clin Pathol 1999;112:391-402.

12 Suzuki K, Nagai K, Yoshida J, et al. Predictors of lymph node and intrapulmonary metastasis in clinical stageIA non-small cell lung carcinoma. Ann Thorac Surg 2001;72:352-356.

13 Luna-More S, Gonzalez B, Acedo C, et al. Invasive micropapillary carcinoma of the breast. A new special type of invasive mammary carcinoma. Pathol Res Pract 1994;190:668-674.

14 Sakamoto K, Watanabe M, De La Cruz C, et al. Primary invasive micropapillary carcinoma of the colon. Histopathology 2005;47:479-484.

15 Perez-Montiel D, Hes O, Michal M, et al. Micropapillary urothelial carcinoma of the upper urinary tract: clinicopathological study of 25 cases. Am J Clin Pathol 2006;126:86-92.

16 Bell KA, Smith Sehdev AE, Kurman RJ, et al. Refined diagnostic criteria for implants associated with ovarian atypical proliferative serous tumors (borderline) and micropapillary serous carcinomas. Am J Surg Pathol 2001;25:419-432.

17 Nagao T, Gaffy TA, Visscher DW, et al. Invasive salivary duct carcinoma. A distinct histologic variant with biologic significance. Am J Surg Pathol 2004;228:319-326.

18 Amin MB, Tamboli P, Merchant SH, et al. Micropapillary component in lung adenocarcinoma: a distinctive histologic feature with possible prognostic significance. Am J Surg Patho 2002;26:358-364.

19 Miyoshi T, Satoh Y, Okumura S, et al. Early-stage lung adenocarcinomas with a micropapillary pattern, a distinct pathologic marker for a significantly poor prognosis. Am J Surg Patho 2003;27:101-109.

20 Tsutsumida $\mathrm{H}$, Nomoto $\mathrm{M}$, Goto $\mathrm{M}$, et al. A micropapillary pattern is predictive of a poor prognosis in lung adenocarcinoma, and reduced surfactant apoprotein $\mathrm{A}$ expression in the micropapillary pattern is an excellent indicator of a poor prognosis. Mod Pathol 2007;20:638-647.

21 Makimoto Y, Nabeshima K, Iwasaki H, et al. Micropapillary pattern: a distinct pathological marker to subclassify tumours with a significantly poor prognosis within small peripheral lung adenocarcinoma ( $\leq 20 \mathrm{~mm}$ ) with mixed bronchioloalveolar and invasive subtypes (Noguchi's type C tumours). Histopathology 2005;46:677-684.

22 Igaki T, Pagliarini RA, Xu T. Loss of cell polarity drives tumor growth and invasion through JNK activation in Drosophila. Curr Biol 2006;16:1139-1146.

23 Frisch SM, Francis H. Disruption of epithelial cell-matrix interactions induces apoptosis. J Cell Biol 1994;124:619-626.

24 Frisch SM, Ruoslahti E. Integrins and anoikis. Cur Opin Cell Biol 1997;9:701-706. 